\title{
A Retrospective Evaluation of Shortened-Duration Oral N-Acetylcysteine for the Treatment of Acetaminophen Poisoning
}

\author{
David P. Betten, MD ${ }^{a}$, Elizabeth E. Burner, $M D^{b}$, Stephen C. Thomas, $M D^{c}$, \\ Christian Tomaszewski, MDd, Richard F. Clark, MD ${ }^{d}$
}

\begin{abstract}
aDepartment of Emergency Medicine, Sparrow Hospital, Michigan State University College of Human Medicine, East Lansing, MI bDepartment of Emergency Medicine, Keck School of Medicine, University of Southern California, Los Angeles, CA

'Department of Emergency Medicine, Naval Hospital, Camp Pendleton, Oceanside, CA

dDepartment of Emergency Medicine, Division of Medical Toxicology, University of California, San Diego, San Diego, CA
\end{abstract}

\begin{abstract}
Introduction: The use of less than the traditional 72-hour course of oral N-acetylcysteine has been an alternative treatment option following potentially toxic acute and chronic acetaminophen ingestions felt to be at low risk of developing hepatotoxicity. While clinical experience with shortened treatment duration is extensive, there are few studies evaluating the effectiveness and extent to which these regimens may be used.

Methods: A large statewide poison center database was reviewed for all acetaminophen exposures involving potentially toxic acute and chronic ingestions, in addition to those taking place at unknown times. Patients were identified who met laboratory criteria for early $\mathrm{N}$-acetylcysteine (NAC) discontinuation (APAP $<10 \mu / \mathrm{mL}$, INR $\leq 1.3$, and AST/ALT $\leq 60$ IU) after a minimum of a $140-\mathrm{mg} / \mathrm{kg}$ oral NAC loading dose and 5 additional 70-mg/kg doses over 20 hours. A further search of the poison center database was conducted for individuals who received shortened-course (20-48 hours) oral NAC treatment who developed subsequent hepatotoxicity or death.

Results: Of 3303 individuals with potentially toxic acetaminophen ingestions, 1932 met criteria for early NAC discontinuation. Mean treatment duration was $36.4 \pm 7.7$ hours (acute $=37.3 \pm 7.6$ hours; chronic $=34.8 \pm 7.4$ hours; unknown $=35.2 \pm 7.6$ hours) The poison center database search identified no short-course eligible subjects who developed subsequent hepatotoxicity or death following $\leq 48$ hours of oral NAC.

Conclusion: Treatment with shortened-course oral NAC in patients meeting criteria for early discontinuation may be an effective treatment option in a sizeable proportion of individuals with potentially toxic acetaminophen ingestions.
\end{abstract}

\section{INTRODUCTION}

Since its introduction to clinical use in the 1950s, acetaminophen has risen to become the most widely-used medication in its class. Given the multitude of over-the-counter and prescription products in which acetaminophen is found, intentional overdoses as well as therapeutic misadventures are not uncommon. In 2006, the American Association of Poison Control Centers (AAPCC) received

Keywords: acetaminophen poisoning, N-acetylcysteine, acetaminophen overdose

Notes: There was no outside funding of any kind used for this study.

The authors have no potential financial conflicts of interest to report.

Corresponding author: David P. Betten, M.D., Sparrow Hospital, Department of Emergency Medicine, 1215 E. Michigan Avenue, Lansing, MI 48912. Email: bettend@msu.edu 
112,809 calls concerning patients who intentionally or accidentally ingested acetaminophen-containing products. Almost 60,000 of these individuals required hospital evaluation, making acetaminophen overdose the most common cause of hospitalization due to ingestion of a pharmaceutical agent [1].

$\mathrm{N}$-acetylcysteine (NAC) has been used to effectively reduce the risk of hepatotoxicity, fulminant hepatic failure, and death following acetaminophen poisoning $[2,3]$. The effectiveness of NAC therapy is believed to lie in its ability to convert acetaminophen's toxic metabolite, N-acetyl-p-benzoquinoneimine (NAPQI), into nontoxic conjugates in addition to other proposed mechanisms such as the improvement of microvascular tone, free radical scavenging, and by disruption of cytokine production [4-6]. The current treatment protocol for oral NAC approved by the United States Food and Drug Administration (FDA) includes a loading dose of $140 \mathrm{mg} / \mathrm{kg}$, followed by 17 doses of $70 \mathrm{mg} / \mathrm{kg}$ every 4 hours, for a total of 72 hours of treatment. Recently, intravenous (IV) NAC has gained FDA approval as a 21-hour continuous infusion for individuals treated within 8-10 hours following an acute overdose. When given within 8 hours following an acute ingestion, NAC is nearly uniformly effective in the prevention of hepatotoxicity (typically defined as AST or ALT $\leq$ 1000 IU) [2,7]. The use of oral and IV NAC is also considered effective in both chronic acetaminophen overdose or suspected supratherapeutic ingestion; however, there is a paucity of literature supporting appropriate NAC treatment indications and duration of treatment in these cases.

Several studies have supported the safety and effectiveness of shortened-duration treatment (20-64 hours) with oral NAC in acute acetaminophen poisoning [8-12]. A survey of poison control directors found that $38 \%$ considered oral NAC treatment of $<72$ hours to be acceptable in certain circumstances [13]. The California Poison Control System (CPCS) has advocated a shortened course of oral NAC (20-36 hours) for more than 15 years in patients considered to be at low risk of developing hepatotoxicity following acetaminophen misuse. While these recommendations have been given with confidence based on our current understanding of the mechanism of acetaminophen-induced hepatotoxicity with no known poor outcomes, follow-up information is often not obtained after hospital discharge. The following retrospective review was performed to further assess the safety and effectiveness of a shortened-course oral NAC treatment protocol in individuals following possible acetaminophen poisoning.

\section{METHODS}

A retrospective evaluation was performed of all cases involving acetaminophen-containing products reported to the CPCS over 5 calendar years (January 1, 1999 to December 31, 2003). Nurses and pharmacists, all of whom are Certified Specialists in Poison Information (CSPIs) by the American Association of Poison Control Centers (AAPCC), were available 24 hours a day, 365 days a year, for poisoning-related treatment recommendations and general information. At the time of each phone call, a computerized file was generated containing general patient information including gender, age, first and last name, time of exposure, and substance ingested. Subsequent communications between CSPIs and the treating health-care team concerning the patient's clinical course were documented in this same computerized database at the time of each phone call.

Typical CPCS treatment guidelines during the study period for acute acetaminophen ingestion included oral NAC administration for patients having serum acetaminophen concentrations $\geq 150$ or $200 \mu \mathrm{g} / \mathrm{mL}$ at 4 hours or the extrapolated equivalent up to 24 hours from the time of ingestion when plotted on the MatthewRumack nomogram. Recommendations were to administer oral NAC as a loading dose of $140 \mathrm{mg} / \mathrm{kg}$, followed by additional 70$\mathrm{mg} / \mathrm{kg}$ doses every 4 hours for a minimum of $20-36$ hours. Differences between the recommended minimum acetaminophen level at which to treat $(150 \mu \mathrm{g} / \mathrm{mL}$ or $200 \mu \mathrm{g} / \mathrm{mL})$ and the recommended treatment duration of shortened-course NAC (SCN) occurred during the study period depending on individual preferences of the CSPI and the 4 divisions of our poison center system. After this minimum number of NAC doses was administered, recommendations were to obtain a repeat serum acetaminophen concentration, liver transaminases, and INR. If serum acetaminophen concentrations were $<10 \mu \mathrm{g} / \mathrm{mL}$, AST and ALT $\leq 60$ $\mathrm{IU}$, and INR $\leq 1.3$, discontinuation of NAC was typically recommended. Individuals presenting with ingestions considered chronic ( $>1$ hour) or at an "unknown time" with any detectable serum acetaminophen present (APAP $\geq 10 \mu \mathrm{g} / \mathrm{mL}$ ) were given recommendations to be treated with NAC for a similar duration (20-36 hours) at standard dosing. An acetaminophen concentration, liver transaminases, and INR were then recommended again after 20 hours of treatment with the same criteria applied as above in acute cases in which the discontinuation of NAC was considered.

Inclusion criteria for this study were all cases reported to the CPCS in which a name, age, and gender was provided for individuals exposed to acetaminophen or acetaminophen-containing products. Acetaminophen ingestions were divided into 3 categories based on time of ingestion: 1) acute, ingested over a $<1$ hour period of time; 2 ) chronic, ingested over a $>1$ hour period of time; or 3) unknown time of ingestion, ingested over an unknown period of time. Acute acetaminophen ingestions were considered "potentially toxic" when plotted at or above the $150-\mu \mathrm{g} / \mathrm{mL}$ line at 4 hours postingestion on the Rumack-Matthew nomogram. These potentially toxic acute acetaminophen ingestions were further divided into "possibly toxic" ingestions (between the $150-\mu \mathrm{g} / \mathrm{mL}$ and $200-\mu \mathrm{g} / \mathrm{mL}$ line on the Matthew-Rumack nomogram) or "probably toxic" ingestions (on or above the $200-\mu \mathrm{g} / \mathrm{mL}$ line on the Matthew-Rumack nomogram). For the purpose of this study, chronic and unknown times of ingestions were considered potentially toxic and were also evaluated for SCN eligibility if the initial acetaminophen concentration was $\geq 10 \mu \mathrm{g} / \mathrm{mL}$ and there was subjective concern by the treating physician and CSPI for possible toxicity requiring NAC treatment.

Patients were excluded for further evaluation in cases in which intravenous NAC was given at any time as well as cases in 
which the treating physician contacting the poison center deemed the ingestion to be nontoxic and provided no NAC therapy. If poison center records made it impossible for study investigators to assess if the ingestion was considered potentially toxic, if acetaminophen levels were not available or NAC was not given within 24 hours from the time of an acute ingestion, or if there was inadequate documentation as to whether the criteria for NAC discontinuation had been met and was or was not a short-course candidate, cases were considered to have insufficient data and were also excluded. Finally, patients discharged from the hospital or sent to a psychiatric hospital with continued oral NAC treatment were also excluded as we were unable to determine ultimate duration of treatment. Upon chart review by study investigators of all included cases, patients were considered eligible for SCN therapy if after a minimum of 6 doses of NAC therapy (20 hours), laboratory studies demonstrated AST and ALT $\leq 60 \mathrm{IU}$, INR $\leq 1.3$, and acetaminophen $<10 \mu \mathrm{g} / \mathrm{mL}$. Patients discharged or cleared medically by the treating physician without having complete laboratory data recorded by the poison specialists were assumed to have had laboratory studies meeting the criteria for early NAC discontinuation. Patients with AST or ALT $>60$ IU or INR $>1.3$ at any time following acetaminophen ingestion were considered to require full-course NAC treatment.

The total duration of NAC treatment was determined from review of the computerized database records given the following assumptions:

1) If a specific time of NAC initiation was not clearly described, it was assumed that NAC was initiated at the time of poison center recommendations.

2) If a precise time of NAC discontinuation was not known, the time at which a telephone call by the poison specialist verified that NAC was no longer being given or the time of hospital discharge (whichever time was earlier) was recorded as the time of NAC discontinuation.

3) Patients reported as being discharged or having NAC discontinued the day previously were assumed to have had NAC discontinued the night before at 10 PM. If treatment was reported as stopped "in the morning," it was assumed NAC was discontinued at $12 \mathrm{PM}$.

4) It was assumed that NAC was not given following hospital discharge unless specifically mentioned by the treating health-care team.

Patient's considered to be eligible based on laboratory criteria for SCN treatment were classified upon chart review by study investigators into 3 categories: 1) SCN received, individuals in which NAC was discontinued at $<48$ hours from the time of treatment onset; 2) full-course NAC received, individuals who were eligible for $\leq 48$ hours of NAC based on the fulfillment of laboratory criteria yet received $>48$ hours of treatment at the treating physician's discretion; 3) unknown-duration NAC, individuals who were eligible for $\leq 48$ hours of treatment, however it is uncertain based on chart review if NAC treatment was discontinued before or after 48 hours from the time of initiation.
A search of the California State Death Statistical Master Database, containing a list of all deaths that occur statewide, was performed to evaluate for the presence of subsequent death for all individuals with acetaminophen exposure found in the CPCS database over the 5-year study period. Individuals found in both databases were considered to have a positive match when the following criteria were met: age matched within 1 year, similar gender, death occurring within 15 days from the time of ingestion, and same name. Investigators were blinded to the listed poison center outcome (death or hospital discharge) at the time of California State Death Statistical Master Database review. Additionally, a search of the poison center database was conducted over the study period to identify individuals who received shortenedcourse (20-48 hours) oral NAC treatment who developed subsequent hepatotoxicity or death.

Data was entered by CSPI's over the 5-year study period into the poison center computerized database. Statistical analysis was performed by taking information pertinent to the study from the database and manually entering this data into a Microsoft Excel spreadsheet (Microsoft Corp., Redmond, WA) for ease of data interpretation. Data from the California State Death Statistical Master Database were evaluated using SAS Statistical software (Version 9.1, SAS Institute, Cary, NC). Categorical data are expressed as the percentage frequency of occurrence. Continuous data are expressed as means with standard deviations (SDs). Statistical differences between means of continuous variables were analyzed with the unpaired 2-tailed student $t$ test. A $p<.05$ was considered significant for all tests. This study was reviewed and approved by the University of California, San Diego, Institutional Review Board.

\section{RESULTS}

We identified a total of 6261 unique cases over the 60-month study period in which acetaminophen was listed as a possibly-ingested substance. Of these, 2958 were excluded from evaluation due to insufficient data, nontoxic ingestions, outpatient NAC therapy, or administration of IV NAC. Of the remaining cases, 1371 required full-course NAC due to abnormal laboratory studies, leaving 1932 eligible for SCN treatment and evaluation (Figure 1). Of individuals receiving $>48$ hours of NAC, death was recorded as the ultimate patient outcome in 111 cases while being followed by the CPCS. A review of all cases involving acetaminophen ingestions in which contact with the statewide poison center was initiated identified no individuals following NAC discontinuation after acute, chronic, or unknown time of ingestions who later returned and developed subsequent hepatotoxicity or death.

Individuals receiving SCN treatment demonstrated a female predominance $(75.5 \%)$ with a clear majority of cases occurring in the 11-20 (41.9\%) and 21-30 (23.8\%) year ranges (Figure 2). The decision among physicians to treat individuals considered to be SCN candidates with $\leq 48$ hours of NAC did not vary significantly based on gender ( $\leq 48$ hours of $\mathrm{NAC}=75.0 \%$ female vs. $>48$ hours 


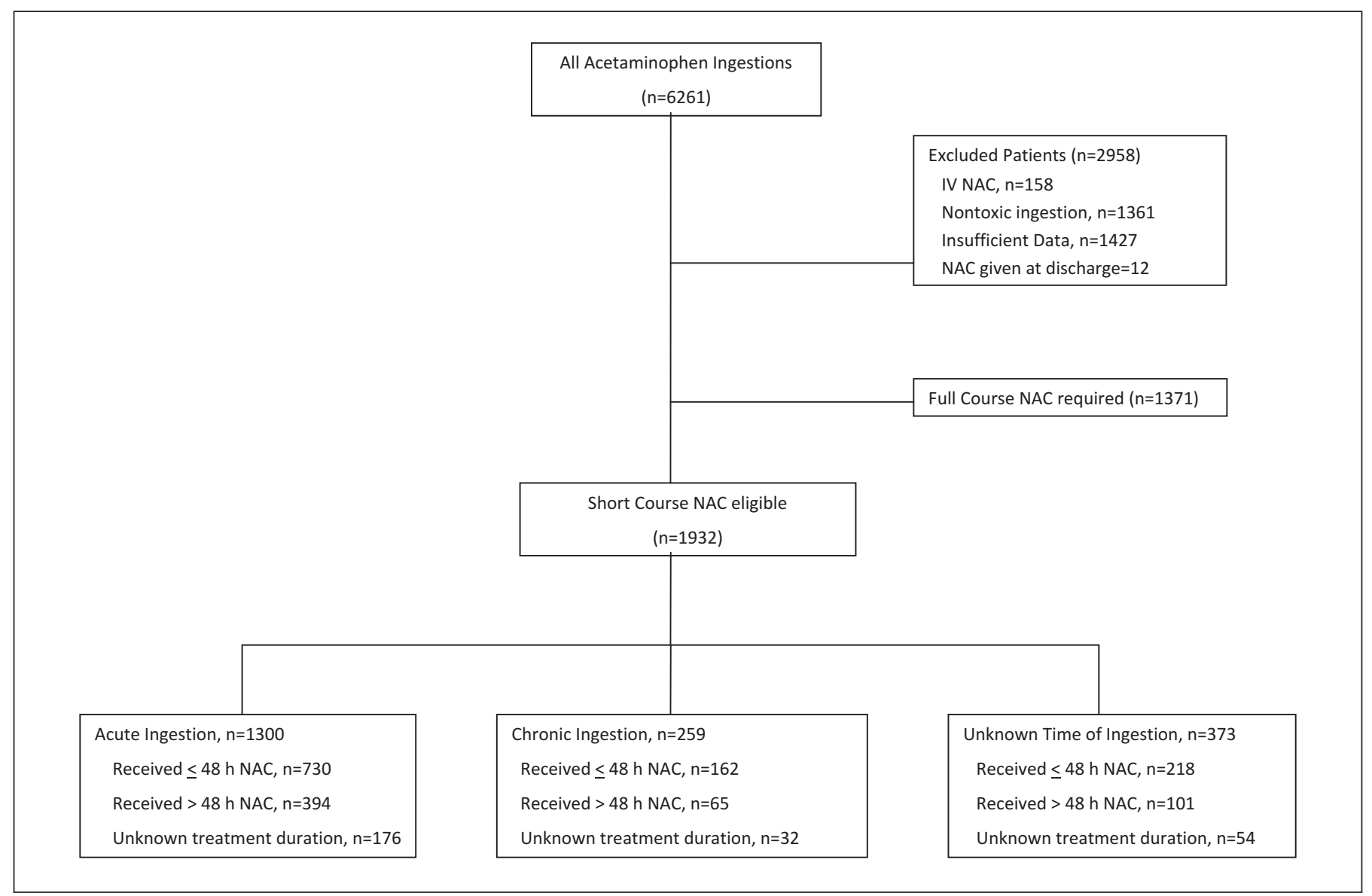

Figure 1: Distribution of short course $\mathrm{N}$-acetylcysteine eligibility and ultimate treatment duration in all individuals with possible acetaminophen ingestion reported over study duration.

of NAC $=75.9 \%$ female, $p=.62$ ) or mean age ( $\leq 48$ hours of NAC $=27.15 \pm 15.2$ years old vs. $>48$ hours of NAC $=27.07 \pm 14.9$ years old; $p=.91$ ).

Mean treatment duration for all individuals treated with $\leq 48$ hours of NAC was $36.42 \pm 7.67$ hours. Time of treatment follow-

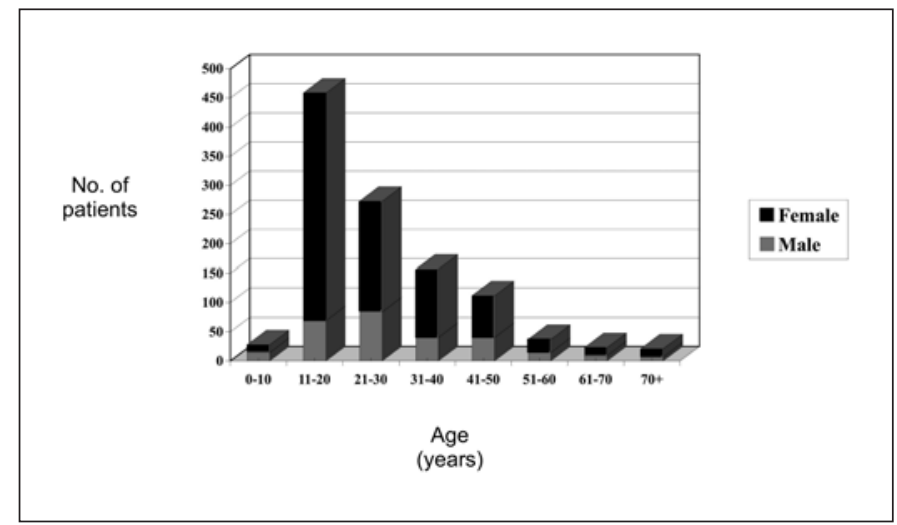

Figure 2: Age and gender distribution of all individuals meeting criteria for shortened duration NAC treatment who were treated with $\leq 48$ hours of NAC. ing acute ingestions (37.27 \pm 7.56 hours) was significantly longer than that in those with chronic ingestion $(34.80 \pm 7.35$ hours, $p=.03)$ and those taking place at unknown times (35.20 $\pm 7.60, p=.04$ ) (Table 1). Implementation of our shortenedcourse oral NAC protocol in all eligible patients could have reduced time of NAC administration by $1-2$ days in $58.5 \%$ of the 3303 individuals with potentially toxic ingestions and sufficient laboratory data available to determine duration of necessary NAC treatment requirements.

The search of the California State Death Statistical Master Database using the previously defined search criteria for all 6261 individuals in which contact with the CPCS was performed identified 113 matches. Of these found individuals, 93 were known to have died while being followed by the CPCS. Eighteen individuals $(16.2 \%)$ who had death recorded in the poison center database as ultimate patient outcome were not found in the State Death Statistical Master Database using our search criteria. Twenty additional cases were identified in the State Death Statistical Master Database that matched patient identifiers in which death was not found to have occurred while being followed by the CPCS. Six of these matches were identified in individuals who were considered eligible for short-course NAC treatment (Figure 3). 
Table 1: Duration of NAC Treatment for Short Course Eligible NAC Candidates Receiving $\leq 48$ Hours of Treatment

\begin{tabular}{|c|c|c|c|}
\hline Ingestion Characteristics & Potential Toxicity & Possible Toxicity & Probable Toxicity \\
\hline \multicolumn{4}{|l|}{ Acute Ingestions } \\
\hline Mean treatment duration $(n=730)$ & $37.27(7.56) \mathrm{h}$ & $\begin{array}{c}36.62(7.70) h \\
(n=323)\end{array}$ & $\begin{array}{c}37.85(7.32) h \\
\quad(n=407)\end{array}$ \\
\hline \multicolumn{4}{|l|}{ Treatment distribution } \\
\hline $20-30 \mathrm{~h}$ & $153(21 \%)$ & $75(23 \%)$ & $78(19 \%)$ \\
\hline$>30-40 \mathrm{~h}$ & $316(43 \%)$ & $134(41 \%)$ & $182(45 \%)$ \\
\hline$>40-48 \mathrm{~h}$ & $261(36 \%)$ & $114(35 \%)$ & $147(36 \%)$ \\
\hline Chronic Ingestions ( $n=162)$ & $34.80(7.35) \mathrm{h}$ & - & - \\
\hline \multicolumn{4}{|l|}{ Treatment distribution } \\
\hline 20-30 hours & $56(34 \%)$ & & \\
\hline$>30-40$ hours & $71(44 \%)$ & & \\
\hline$>40-48$ hours & $35(22)$ & & \\
\hline Unknown Time of Ingestion $(n=218)$ & $35.20(7.60) h$ & - & - \\
\hline \multicolumn{3}{|l|}{ Treatment distribution } & \\
\hline$>30-40 \mathrm{~h}$ & $96(44 \%)$ & & \\
\hline$>40-48 \mathrm{~h}$ & 59 (27\%) & & \\
\hline
\end{tabular}

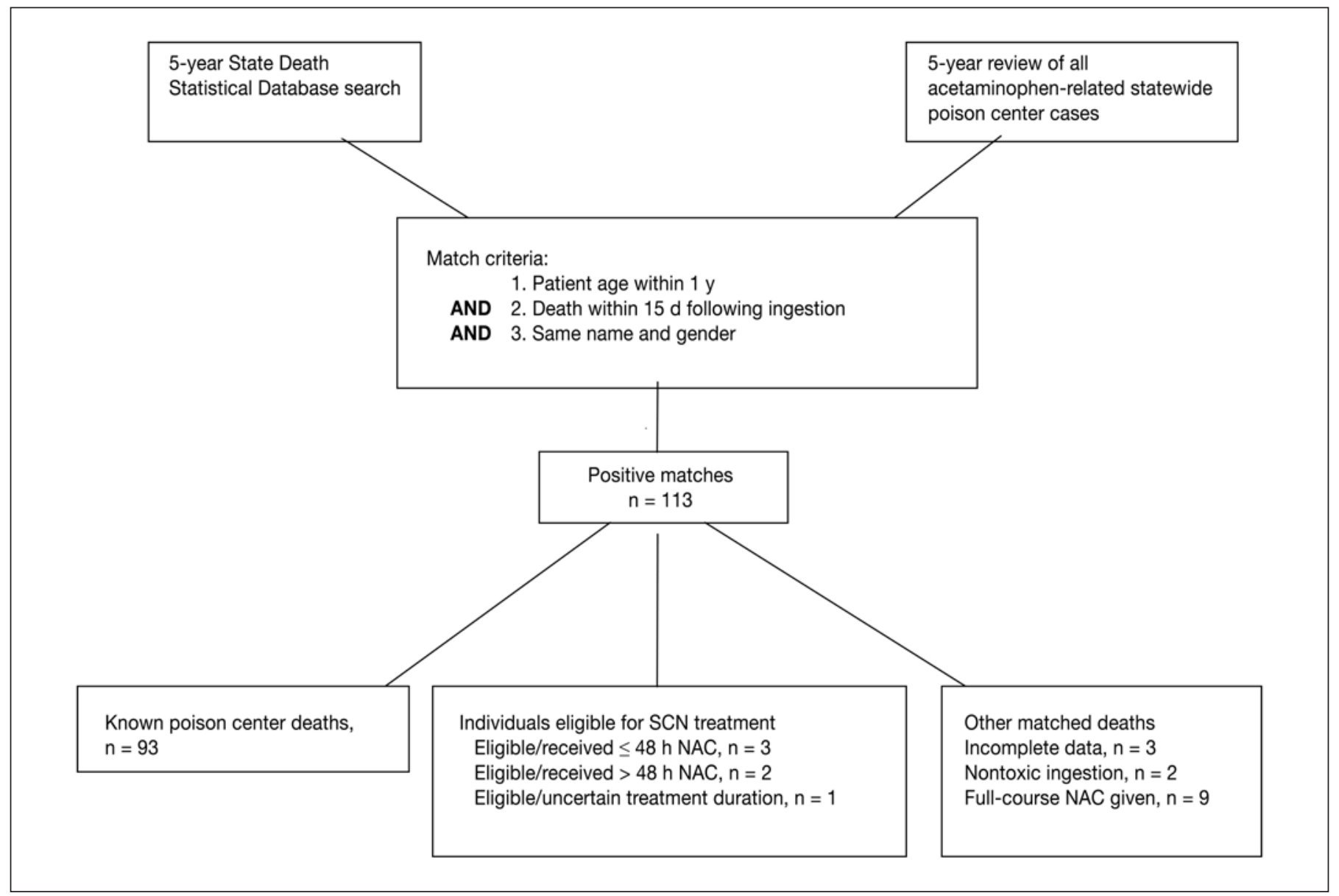

Figure 3: Search criteria utilized and identification of death in all individuals in which contact with the statewide poison center was initiated for acetaminophen-related ingestions over the 5-year study period. 
Table 2: Deaths in Individuals Eligible for SCN Treatment

\begin{tabular}{lccc}
\hline Age/Gender & $\begin{array}{c}\text { Acute or } \\
\text { Chronic Ingestion }\end{array}$ & $\begin{array}{c}\text { Received SCN } \\
\text { Treatment? }\end{array}$ & Cause of Death \\
\hline 27 female & Acute & Unknown & Auto accident \\
\hline 39 male & Chronic & Yes & Accidental poisoning \\
\hline 49 male & Acute & $>48 \mathrm{~h}$ & Atherosclerotic heart disease \\
\hline 52 male & Chronic & $>48 \mathrm{~h}$ & Atherosclerotic heart disease \\
\hline 65 male & Acute & Yes & Intentional self-harm by hanging \\
\hline 65 female & Chronic & Yes & Aspiration pneumonitis \\
\hline
\end{tabular}

"Accidental poisoning" was recorded as cause of death in 1 of these 6 cases (Table 2). Death occurred in a 39-year-old male with a chronic acetaminophen ingestion with an initial acetaminophen level of $38 \mu \mathrm{g} / \mathrm{mL}$ and "normal" liver function tests and coagulation studies the afternoon of presentation, at which time NAC was initiated. The following morning, blood acetaminophen was measured and "nondetectable" (AST = $18 \mathrm{IU}$, ALT = $27 \mathrm{IU}$, INR = 1.0), and NAC was reported to be "continued." No further laboratory studies were reported. The treating medical team medically cleared the patient and discontinued NAC by 46 hours from the time of initial treatment. The CPCS received no further information concerning this individual. Twelve days following the initial ingestion the patient died of an "accidental poisoning," as reported on the California State Death Statistical Master Database.

\section{DISCUSSION}

The use of shortened-duration oral N-acetylcysteine therapy when applied to an acceptable patient subset considered at low risk of developing hepatotoxicity has been used among some members of the medical toxicology community for years [8-12]. The rationale supporting a shortened-course treatment duration for acetaminophen-poisoned patients is based on the traditional understanding of acetaminophen-induced toxicity. NAPQI, acetaminophen's toxic metabolite, continues to accumulate and damage hepatocytes as long as acetaminophen is present in amounts that overwhelm endogenous glutathione stores. N-acetylcysteine's ability to detoxify NAPQI would support continued NAC treatment until acetaminophen is no longer present as it may be presumed that only then will there be an absence of further NAPQI production. Abnormal hepatic laboratory studies implying a NAPQI-induced insult may justify additional treatment as well given the possible benefits of NAC in attenuating the cascade of inflammatory mediators, free-radical formation, and neutrophil-induced inflammation that may ultimately result in further hepatic damage $[3,5,14]$.

There are limitations to a shortened-duration treatment protocol and earlier NAC discontinuation. The possibility of a delay between NAPQI production and hepatocyte damage as evidenced by transaminase and INR elevation may occur [15]. The duration of time needed for the development of NAPQI-induced hepatocyte damage to occur has not been clearly defined, raising the possibility of a mild, non-clinically significant elevation in laboratory studies even after treatment endpoints (APAP $<10 \mu \mathrm{g} / \mathrm{mL}$ and AST/ALT $\leq 60 \mathrm{IU}, \mathrm{INR} \leq 1.3$ ) have been met. Additionally, our study's endpoint of treatment included an acetaminophen level $<10 \mu \mathrm{g} / \mathrm{mL}$, the lowest level reported in many laboratories. While low, a concentration below $10 \mu \mathrm{g} / \mathrm{mL}$ may not be the same as no acetaminophen present, and small amounts of remaining drug may initiate some degree of NAPQI-induced hepatocyte damage should the body's endogenous detoxifying mechanisms be near depletion.

A further difficulty in the use of laboratory values in the normal range is that this represents a normal range across a population and may not take into account individual baseline values. For example, an ALT $=60 \mathrm{IU}$ and INR $=1.3 \mathrm{in}$ an individual with baseline values of ALT $=10 \mathrm{IU}$ and INR $=1.0$ may be evidence of early-onset mild acetaminophen-induced toxicity that may be followed by further transaminase and INR elevations after NAC discontinuation. Despite limitations in these defined endpoints, it is our belief that it is highly unlikely that hepatotoxicity (typically defined as AST or ALT >1000 IU), fulminant hepatic failure, or death would occur in individuals fulfilling this study's laboratory criteria for NAC discontinuation. Although a mild transaminitis may occur even in patients in whom NAC is begun early and continued for 72 hours, the typical course in these cases is full hepatocyte recovery. A further limitation includes the fact that many ingestions evaluated in this study involved polysubstance exposures, leading to the possibility of other substances that may have contributed to laboratory study abnormalities.

This study represents, to our knowledge, the first large-scale review of shortened-duration NAC treatment in potentially toxic, nonacute acetaminophen exposures. Endpoints of treatment similar to those in our study have been suggested in individuals with chronic poisoning and ingestions taking place at unknown times; however, these types of ingestions remain inadequately studied. These patient subsets, for which the Matthew-Rumack nomogram is not applicable and current FDA guidelines for both oral and IV NAC do not address, in our review made up a sizeable minority [632/1931(32.7\%)] of the total number of patients 
that were considered potentially toxic and met criteria for early NAC discontinuation.

Our study is limited in the fact that follow-up laboratory studies were not obtained after criteria for NAC discontinuation were met. We therefore are unable to evaluate whether a delayed rise in transaminase levels or INR may have occurred. Should an individual have returned to the hospital with laboratory or clinical evidence of hepatotoxicity, this may not have been reported to the poison center. Notification of a return visit would be entirely dependent on the primary treating health-care providers' willingness to do so. Full laboratory criteria, including all required endpoints of treatment, were not recorded in the poison center computerized database in many individuals in which NAC was discontinued. We speculate this to be due to laboratory studies not being reported by hospital personnel or specific results not entered by CSPIs rather than that they were not obtained. Endpoints of treatment are routinely reviewed at the time of initial and subsequent CPCS contact. The duration of NAC treatment was likely overestimated in many cases, as a precise time of NAC discontinuation was frequently not recorded by CSPIs. Similarly, in some cases the exact time following acetaminophen ingestion at which NAC was begun was not available, thus preventing precise analysis of treatment duration based on the time of initial treatment. It was felt more appropriate to overestimate the time of treatment while reviewing data so that conclusions drawn from this study would be based on more conservative, longertreatment durations than what may have actually occurred.

A total of 111 individuals were known to have died while being followed by the poison center. All of the individuals who demonstrated laboratory evidence of heptatotoxicity received continuous treatment with oral or IV NAC until death. Using search criteria as defined in the Methods section of this article, $83.8 \%$ (93/111) of individuals who were known to have died were identified in the State Death Statistical Master Database. It is assumed that the 18 individuals who were not identified, yet were known to have died, had either inaccurately reported ages or incorrect spellings of names by either hospital staff or poison center personnel. Given the lack of sensitivity of this statewide death index search in patients with known death, we are unable to say with certainty whether those discharged after NAC discontinuation ultimately had death as a possible outcome. Death following acetaminophen poisoning is an unusual outcome and death or hepatic failure in any individual who received shortened-duration NAC would represent a failure of this treatment protocol. While we believe these outcomes to be unlikely, the sensitivity of our poison center search matched with the State Death Statistical Master Database does not allow us to reach this conclusion.

It is uncertain whether the 6 deaths in individuals considered eligible for SCN treatment found in the State Death Statistical Master Database are different individuals with similar patient identifiers (name and age) or the same individual treated by poison center personnel. The cause of death reported in 5 of 6 cases suggests a clear cause other than acetaminophen poisoning. The remaining individual who died of "intentional poisoning" fol- lowing a chronic supratherapeutic ingestion should be noted to have died 12 days following the initial presentation and 10 days after hospital discharge. Death occurring this late after an acetaminophen exposure falls well beyond a typical time course of death following acetaminophen poisoning. A second overdose following initial discharge seems plausible and one possibility.

Specific limitations based on our study design in patient follow-up and a lack of sensitivity in our search of the Statewide Death Statistical Master Database unfortunately hamper our ability to reach these conclusions with certainty. The U.S. FDA-guided landmark article by Smilkstein et al. and subsequent approval of oral NAC as a full 3-day course of treatment appears to be based on the assumption of prolonged acetaminophen halflives of greater than 12 hours $[2,16]$. These prolonged half-lives are atypical and would be expected to be found only in those individuals following delayed or absent NAC treatment in which acetaminophen-induced liver dysfunction has already taken place rather than in those low-risk individuals for whom treatment was initiated earlier or in those with ingestions of borderline toxicity. Shorter IV NAC protocols (20-48 hours) implemented outside the United States at a similar time as the 72-hour oral NAC protocol treated for a duration more adequate for a more typical conservative estimate of an acetaminophen half-life of $<4$ hours. In this case, after 5 half-lives, or 20 hours, nearly all acetaminophen would be nearly fully metabolized unless absorption is delayed, metabolism is impaired by damaged hepatocytes, or metabolic enzymes are saturated. Intravenous treatment protocols of 20-48 hours have been in use for over two decades in Europe, Canada, and Australia with apparent success [7,17-20]. Widespread clinical experience with these treatment regiments offers further evidence for what is undoubtedly a very effective treatment option. The success of shortened-duration IV NAC protocols would appear to lend some additional support to shorter-duration oral $\mathrm{N}$-acetylcysteine. After absorption, oral NAC is pharmacologically identical to intravenous NAC, and the large amount of oral NAC traditionally given in the first 20 hours $(490 \mathrm{mg} / \mathrm{kg})$ is assumed to be more than sufficient to replenish glutathione stores depleted by NAPQI production.

Shortened-course oral NAC has been used in many acetaminophen-poisoned patients for decades with apparent clinical success. It has yet to succeed, however, in becoming fully accepted in the medical community as an effective treatment option in those patients considered to be in a low-risk patient subset following acetaminophen ingestions. This retrospective review, other recently-published reports, and years of widespread clinical experience with shortened oral NAC protocols suggest that a shortened-course oral NAC therapy may be effective and appropriate for a large portion of acetaminophen-poisoned patients.

\section{REFERENCES}

1. Bronstein AC, Spyker DA, Cantilena LR, et al. 2006 Annual report of the American Association of Poison Control 
Centers' National Poison Data System (NPDS). Clin Toxicol. 2007;45:815-917.

2. Smilkstein MJ, Knapp GL, Fulig KW, et al. Efficacy of oral $\mathrm{N}$-acetylcysteine in the treatment of acetaminophen overdose: analysis of the National Multicenter Study (1976 to 1985).

N Engl J Med. 1988;319:1557-1562.

3. Keays R, Harrison PM, Wendon JA, et al. Intravenous acetylcysteine in paracetamol induced fulminant hepatic failure: a prospective controlled trial. BMJ. 1991;303: 1026-1027.

4. James LP, Farrar HC, Darville Rl, et al. Elevation of serum interleukin 8 levels in acetaminophen overdose in children and adolescents. Clin Pharmacol Ther. 2001;70:280-286.

5. Auroma OI, Halliwell B, Hoey BM, et al. The antioxidant action of $\mathrm{N}$-acetycysteine: its reaction with hydrogen peroxide, hydroxyl radical, superoxide, and hypochlorous acid. Free Radic Biol Med. 1989;6:593-597.

6. Jollow DJ, Mitchell FR, Potter WZ, et al. Acetaminopheninduced hepatic necrosis, II: role of covalent binding in vivo. J Pharmacol Exp Ther. 1973;187:195-202.

7. Prescott LF, Illingworth RN, Critchley JA, et al. Intravenous $\mathrm{N}$-acetylcysteine: the treatment of choice for paracetamol poisoning. BMJ. 1979;2:1097-1100.

8. Woo OF, Mueller PD, Olson KR, et al. Shorter duration of oral N-acetylcysteine therapy for acute acetaminophen overdose. Ann Emerg Med. 2000;35:363-368.

9. Yip L, Dart RC. A 20-hour treatment for acute acetaminophen overdose. N Engl J Med. 2003;348:2471-2472.

10. Tsai CL, Chang ST, Eng TI, et al. A patient-tailored $\mathrm{N}$-acetylcysteine protocol for acute acetaminophen intoxication. Clin Ther. 2005;27:336-341.
11. Betten DP, Cantrell FL, Thomas SC, et al. A prospective evaluation of shortened course oral $\mathrm{N}$-acetylcysteine for the treatment of acute acetaminophen poisoning. Ann Emerg Med. 2007;50:272-279.

12. Dart RC, Rumack BH. Patient-tailored acetylcysteine administration. Ann Emerg Med. 2007;50:280-281.

13. Kozer E, McGuigan M. Treatment strategies for early presenting acetaminophen overdose: a survey or medical directors of poison center in North America and Europe. Hum Exp Toxicol. 2002;21:123-127.

14. Ramaiah SK, Jaeschke H. Role of neutrophils in the pathogenesis of acute inflammatory liver injury. Toxicol Pathol.

2007;25:757-766.

15. Singer AJ, Carracio TR, Mofenson HC. The temporal profile of increased transaminase levels in patients with acetaminophen induced liver injury. Ann Emerg Med. 1995;26:49-53.

16. Rumack BH. Acetaminophen hepatotoxicity: the first 35 years. J Toxicol Clin Toxicol. 2002;40:3-20.

17. Smilkstein MJ, Bronstein AC, Linden C, et al. Acetaminophen overdose: a 48-hour intravenous $\mathrm{N}$-acetylcysteine treatment protocol. Ann Emerg Med. 1991;20:1058-1063.

18. Sivilotti ML, Yarema MC, Juurlink DN, et al. A risk quantification instrument for acute acetaminophen overdose patients treated with N-acetylcysteine. Ann Emerg Med. 2005;46:263-271.

19. Buckley NA, Whyte IM, O'Connell DL, et al. Oral or intravenous $\mathrm{N}$-acetylcysteine: which is the treatment of choice for acetaminophen (paracetamol) poisoning? J Toxicol/Clin Toxicol. 1999;37:759-767.

20. Parker D, White JP, Paton D, et al. Safety of late acetylcysteine treatment in paracetamol poisoning. Hum Exp Toxicol. 1990;13:25-27. 